Bull. Austral. Math. Soc.

30D05, 32н04

VOL. $61(2000) \quad[1-9]$

\title{
BOREL DIRECTIONS AND ITERATED ORBITS OF MEROMORPHIC FUNCTIONS
}

\author{
JiANYONG QIAO
}

For transcendental meromorphic functions of finite order, we prove that there exist iterated orbits which tend to the Borel directions. This gives a relation between the value distribution theory and the iteration theory of meromorphic functions.

\section{INTRODUCTION}

Suppose $f: \mathbf{C} \rightarrow \overline{\mathbf{C}}$ is a transcendental meromorphic function. If for any $\varepsilon>0, f$ takes every complex value $a$ infinitely many times on the region: $\left|\arg z-\theta_{0}\right|<\varepsilon$, with at most two exceptional values $a \in \overline{\mathbf{C}}$, then the ray $\arg z=\theta_{0}$ is said to be a Julia direction of $f(z)$. Furthermore, if for any $\varepsilon>0$,

$$
\varlimsup_{r \rightarrow \infty} \frac{\log n\left(r, \theta_{0}, \varepsilon, f=a\right)}{\log r} \geq \omega>0,
$$

with at most two exceptional values of $a \in \overline{\mathbf{C}}$, where $n\left(r, \theta_{0}, \varepsilon, f=a\right)$ is the number of roots of $f(z)=a$ on the region: $|z|<r$ and $\left|\arg z-\theta_{0}\right|<\varepsilon$, then the ray $\arg z=\theta_{0}$ is said to be a Borel direction of order at least $\omega$. These are fundamental concepts in value distribution theory [5].

In this note, we deal with the problem: Can we choose an iterated orbit such that it approximates to the Borel directions? Define

$$
I(f)=\left\{z \in \mathbf{C} \mid f^{n}(z) \neq \infty \text { for all } n \text { and } f^{n}(z) \rightarrow \infty \text { as } n \rightarrow \infty\right\},
$$

where $f^{n}$ is the n-th iterate of $f$, that is, $f^{0}(z)=z$ and $f^{n}(z)=f \circ f^{n-1}(z)$ for $n \geq 1$. $f^{n}(z)$ is defined for all $z \in \mathbf{C}$ except for a countable set which consists of the poles of $f, f^{2}, \cdots, f^{n-1}$. Obviously, the forward orbit $O^{+}(a)=\left\{f^{n}(a) \mid n \geq 0\right\}$ is an infinite set if $a \in I(f)$. We want to find a point $a \in \mathbf{C}$ such that $a \in I(f)$ and each limiting direction of $\mathrm{O}^{+}(a)$ (that is, a limit of $\left\{\arg z \mid z \in O^{+}(a)\right\}$ ) is a Borel direction of $f$. By $J(f)$ denote the Julia set of $f$ which is the closure of the set of the repelling periodic points; its complement $F(f)$ is the Fatou set (see [2]). In this note we shall prove

THEOREM 1. Let $f(z)$ be a transcendental meromorphic function, then $I(f) \cap$ $J(f) \neq \emptyset$.

Received 16th February, 1999

Project supported by the National Natural Science Foundation of China.

Copyright Clearance Centre, Inc. Serial-fee code: 0004-9727/00 \$A2.00+0.00. 
REMARK. Eremenko [3] has proved this result for transcendental entire functions.

THEOREM 2. Let $f(z)$ be a transcendental meromorphic function of finite order, the lower order $\mu>0$. Then there exists a point $a \in I(f) \cap J(f)$ such that each limiting direction of $\mathrm{O}^{+}(a)$ is a Borel direction of order at least $\mu$.

REMARK. It is well known that there exist transcendental meromorphic functions of lower order zero which don't have a Julia direction [5].

Since the backward orbit $O^{-}(a)=\left\{z \mid f^{n}(z)=a\right.$ for some $\left.n\right\}$ is dense on $J(f)$ for every point $a \in J(f)$ with at most one exceptional point [2], we easily have

COROLLARY. Let $f(z)$ be a transcendental meromorphic function of finite order, the lower order $\mu>0$. Then there is a dense subset $I_{B}$ of $J(f)$ such that, for $a \in$ $I_{B}, O^{+}(a)$ tends to infinity and each limiting direction of $O^{+}(a)$ is a Borel direction of order at least $\mu$.

\section{THE PROOF OF THEOREM 1}

In order to prove Theorem 1, we need the following lemma:

LEmma 1. [1] Suppose, in a domain $D$, the analytic functions $f$ of the family $G$ omit the values 0,1 , and $H$ is a compact subset of $D$ on which the functions all satisfy $|f(z)| \geq 1$. Then there exist constants $k$, $t$, dependent only on $H$ and $D$, such that for any $z, z^{\prime} \in H$ and any $f \in G$ we have $\left|f\left(z^{\prime}\right)\right|<k|f(z)|^{t}$.

THE PRoOF of Theorem 1: We distinguish the following two cases:

A. $f(z)$ has infinitely many poles. Let $a_{0}$ be a pole of $f(z)$, then there exists a constant $R>1$ such that $f\left(V\left(a_{0}\right)\right) \supset\{z \cdot|z|>R\}$, where $V(\eta)=\{z|| z-\eta \mid<1\}$. Choose a pole $a_{1} \in\{z|| z \mid>R+2\}$, then $f\left(V\left(a_{0}\right)\right) \supset \overline{V\left(a_{1}\right)}$. Since $a_{1}$ is also a pole, there exists a constant $l_{1} \geq 2$ such that $f\left(V\left(a_{1}\right)\right) \supset\left\{z|| z \mid>R^{l_{1}}\right\}$. By repeating this construction, we obtain a sequence of disks $V\left(a_{j}\right)\left(a_{j}\right.$ is a pole) such that $V\left(a_{j}\right) \rightarrow \infty$ and

$$
f\left(V\left(a_{j}\right)\right) \supset \overline{V\left(a_{j+1}\right)} \quad(j=0,1,2, \ldots) .
$$

It is obvious that there exists a sequence of domains $B_{j} \subset V\left(a_{0}\right)$ such that $\overline{B_{j+1}} \subset B_{j}$ and $f^{j}\left(B_{j}\right)=V\left(a_{j}\right)$. For a point $a \in \bigcap_{j=1}^{\infty} \overline{B_{j}}$, we have $a \in I(f)$. Since $a_{j}$ is a pole, then $V\left(a_{j}\right) \cap J(f) \neq \emptyset$, and thus $B_{j} \cap J(f) \neq \emptyset$ for all $j[2]$. So we have $a \in I(f) \cap J(f)$.

B. $f(z)$ has only finitely many poles. By Mittag-Leffler's theorem,

$$
f(z)=g(z)+\sum_{j=1}^{m} P_{j}\left(\frac{1}{z-a_{j}}\right)
$$


where $g(z)$ is a transcendental entire function, $a_{j}(j=1, \cdots, m)$ are $m(<\infty)$ distinct poles of $f(z)$, and $P_{j}$ is a polynomial with $P_{j}(0)=0$. For a transcendental entire function $g(z)$, Eremenko [3] proved: there exist a sequence of positive numbers $r_{j} \rightarrow \infty$, a constant $b>1$ and a sequence of domains $\sigma_{j} \subset\left\{z\left|r_{j} / b<\right| z \mid<b r_{j}\right\}$ such that

$$
g\left(\sigma_{j}\right) \supset\left\{z\left|\frac{1}{b_{1}} r_{j+1}<\right| z \mid<b_{1} r_{j+1}\right\} \quad(j=1,2, \cdots),
$$

where $b_{1}>b$ is a constant. For a constant $b_{2} \in\left(b, b_{1}\right)$, by (1) and (2) we deduce that there exists $j_{0}>0$ such that

$$
f\left(\sigma_{j}\right) \supset\left\{z\left|\frac{1}{b_{2}} r_{j+1}<\right| z \mid<b_{2} r_{j+1}\right\} \supset \sigma_{j+1}
$$

when $j \geq j_{0}$. So there exists a sequence of domains $B_{p} \subset \sigma_{j_{0}}$ such that

$$
f^{p}\left(B_{p}\right)=\sigma_{j_{0}+p}, \quad \overline{B_{p+1}} \subset B_{p}, \quad p=1,2, \cdots .
$$

It follows that $\bigcap_{p=1}^{\infty} \overline{B_{p}} \subset I(f)$, thus $I(f) \neq \emptyset$.

If $\left(\bigcap_{p=1}^{\infty} \widetilde{B_{p}}\right) \cap J(f) \neq \emptyset$, we have $I(f) \cap J(f) \neq \emptyset$. Below we suppose $\left(\bigcap_{p=1}^{\infty} \overline{B_{p}}\right) \cap$ $J(f)=\emptyset$, then there exists $p_{0} \geq 1$ such that $B_{p} \subset F(f)$ when $p \geq p_{0}$. By (3) and (4) we have

$$
\left\{z\left|\frac{1}{b_{2}} r_{j}<\right| z \mid<b_{2} r_{j}\right\} \subset F(f)
$$

when $j \geq p_{0}+j_{0}+1$.

Now, we prove that $F(f)$ has only bounded components: Assume $D$ is an unbounded component of $F(f)$. By (3) and (5) we know that $f(D) \subset D, f^{n}(z) \rightarrow \infty$ for $z \in D$ and $\overline{\sigma_{j}} \subset D$ when $j \geq p_{0}+j_{0}+1$. Put

$$
H=\overline{\sigma_{p_{0}+j_{0}+1} \cup f\left(\sigma_{p_{0}+j_{0}+1}\right)}
$$

then $H \subset D$. Without loss of generality, we may assume $0,1 \in J(f)$ and $\left|f^{n}(z)\right| \geq 1$ on $H$ for all $n$. By Lemma 1, for any $z^{\prime} \in \sigma_{p_{0}+j_{0}+1}$ we have

$$
\left|f^{n+1}\left(z^{\prime}\right)\right|<k\left|f^{n}\left(z^{\prime}\right)\right|^{t}, \quad n=1,2, \cdots,
$$

where $k$ and $t$ are two constants. Put $\Omega=\bigcup_{n=0}^{\infty} f^{n}\left(\sigma_{p_{0}+j_{0}+1}\right)$, then for any $z \in \Omega$, there exist a point $z^{\prime} \in \sigma_{p_{0}+j_{0}+1}$ and a natural number $n$ such that $f^{n}\left(z^{\prime}\right)=z$. By (6) we get

$$
|f(z)|<k|z|^{t}, \quad z \in \Omega
$$


Noting $\Omega \supset\left\{z\left|r_{j} / b<\right| z \mid<b r_{j}\right\}$ for sufficiently large $j$, we have

$$
M\left(r_{j}, g\right)=M\left(r_{j}, f\right)+o(1)=O\left(r_{j}^{t}\right) \quad\left(r_{j} \rightarrow \infty\right)
$$

This contradicts the transcendence of $g(z)$. Therefore $F(f)$ has only bounded components.

Denote the component of $F(f)$ containing $B_{p_{0}}$ by $D_{0}$. Since $B_{p_{0}} \cap I(f) \neq \emptyset$, so $f^{n}(z) \rightarrow \infty$ for $z \in D_{0}$. It follows from (5) and the boundedness of $D_{0}$ that $f^{n}\left(\partial D_{0}\right) \rightarrow \infty$, and thus $\partial D_{0} \subset I(f) \cap J(f)$. The proof of Theorem 1 is complete. $]$

\section{ThE PROOF OF THEOREM 2}

Denote the Nevanlinna characteristic function of $f(z)$ by $T(r, f)$ [5]. Since $f$ is of positive lower order and finite order, there exists a constant $\alpha>1$ such that $T(2 r, f)<T^{\alpha}(r, f)$ for sufficiently large $r$. Therefore, Theorem 2 is the corollary of the following result:

THEOREM 3. Let $f(z)$ be a transcendental meromorphic function of lower order $\mu \in(0, \infty)$. If

$$
\varlimsup_{r \rightarrow \infty} \frac{\log T(2 r, f)}{\log T(r, f)}<\infty,
$$

then there exists a point $a \in I(f) \cap J(f)$ such that each limiting direction of $O^{+}(a)$ is a Borel direction of order at least $\mu$.

In order to prove Theorem 3 , we need the following lemmas:

LEMma 2. [5] Let $f$ be a transcendental meromorphic function. If $R$ is suffciently large to satisfy

$$
T(R, f) \geq \max \left\{240, \frac{240 \log (2 R)}{\log k}, 12 T(r, f), \frac{12 T(k r, f)}{\log k} \log \frac{2 R}{r}\right\}
$$

then there exists a point $z_{j}$ lying in $r<|z|<2 R$ such that in the domain

$$
\Gamma: \quad\left|z-z_{j}\right|<\frac{4 \pi}{q}\left|z_{j}\right|
$$

$f$ takes every complex value at least

$$
n=c^{*} \frac{T(R, f)}{q^{2}\left(\log \frac{r}{R}\right)^{2}}
$$


times except for those complex values which can be contained in two spherical disks each with radius $e^{-n}$, where $k>1$ is a constant, $q$ is a sufficiently large integer, and $c^{*}>0$ is an absolute constant. The disk $\Gamma$ is called a filling disk of $f(z)$.

LEMMA 3. [4] Let $T(r)$ be a positive, increasing and continuous function, and $T(r) \rightarrow+\infty(r \rightarrow+\infty)$. If

$$
\varlimsup_{r \rightarrow \infty} \frac{\log T(r)}{\log r} \leq \nu<+\infty
$$

then for any two numbers $\tau_{1}>1, \tau_{2}>1$, the lower logrithmic density of the set $\left\{r \mid T\left(\tau_{1} r\right) \leq \tau_{2} T(r)\right\}$ is not less than $1-\left(\nu \log \tau_{1}\right) /\left(\log \tau_{2}\right)$.

Lemma 4. Let $T(r)$ be a positive, increasing and continuous function, and $T(r) \rightarrow+\infty(r \rightarrow+\infty)$. If

$$
\lim _{r \rightarrow \infty} \frac{\log T(r)}{\log r} \geq \omega>0
$$

where $\tau_{1}>1, \tau_{2}>1$ are two constants satisfying $\tau_{2}<\tau_{1}^{\omega}$, then for any constant $m>1 /\left(1-\left(\log \tau_{2}\right) /\left(\omega \log \tau_{1}\right)\right)$, there exists a constant $R_{0}>0$ such that

$$
\left\{t \mid \tau_{2} T(t) \leq T\left(\tau_{1} t\right)\right\} \cap\left[r, T^{-1}\left(T^{m}(r)\right)\right] \neq \emptyset
$$

when $r>R_{0}$.

The proof of Lemma 4: Put $s=T(r), T_{0}(s)=T^{-1}(s)$. Then $T_{0}(s)$ is a positive, increasing and continuous function, and $T_{0}(s) \rightarrow+\infty(s \rightarrow+\infty)$. Obviously,

$$
\varlimsup_{s \rightarrow \infty} \frac{\log T_{0}(s)}{\log s} \leq \frac{1}{\omega}<+\infty
$$

By Lemma 3,

$$
\text { lower-logdens }\left\{s \mid T_{0}\left(\tau_{2} s\right) \leq \tau_{1} T_{0}(s)\right\} \geq 1-\frac{\log \tau_{2}}{\omega \log \tau_{1}}
$$

Therefore, there exists $s_{0} \in\left[s, s^{m}\right]$ such that $T_{0}\left(\tau_{2} s_{0}\right) \leq \tau_{1} T_{0}\left(s_{0}\right)$ for sufficiently large $s$. Put $r_{0}=T^{-1}\left(s_{0}\right)$, then $r_{0}=T_{0}\left(s_{0}\right), T\left(r_{0}\right)=s_{0}$. Thus $\tau_{2} T\left(r_{0}\right) \leq T\left(r_{1} r_{0}\right)$. Since $r_{0} \geq r, T\left(r_{0}\right)=s_{0} \leq s^{m}=T^{m}(r)$, we deduce $r_{0} \in\left[r, T^{-1}\left(T^{m}(r)\right)\right]$. The proof of Lemma 4 is complete.

The PROOF OF Theorem 3: Choose two constants $k>1$ and $\tau_{1}>1$ such that

$$
\frac{12}{\log k} \log \left(2 k \tau_{1}\right)<\tau_{1}^{\mu}
$$


Put

$$
\tau_{2}=\frac{12}{\log k} \log \left(2 k \tau_{1}\right) \text { and } \alpha=\varlimsup_{r \rightarrow \infty} \frac{\log T(2 r, f)}{\log T(r, f)} .
$$

Choose a natural number $m$ such that

$$
m>\max \left(\frac{1}{1-\left(\log \tau_{2}\right) /\left(\mu \log \tau_{1}\right)}, 2 \alpha\right) .
$$

For convenience, we put $T(r, f)=T(r)$. It is obvious that there exists a constant $M_{0}>0$ such that

$$
\begin{gathered}
M_{0}>\max \left\{R_{0}, e^{8 \pi}\right\}, \\
T(r)>\max \left\{\frac{1}{K}(\log r)^{2 m^{4 p+1}+2}, \frac{240 \log (2 r)}{\log k}\right\}, \\
T(2 r, f)<T^{2 \alpha}(r, f), \\
c \cdot c^{*} \frac{\tau_{1}^{\mu / 2} r^{\mu / 4}}{\left(\log \left(k \tau_{1}\right) \log r\right)^{2}}>1
\end{gathered}
$$

when $r \geq M_{0}$, where $R_{0}>0$ is the constant stated in Lemma $4, c^{*}>0$ is the constant stated in Lemma 2, and

(12) $c=\frac{1}{1+9 \tau_{1}^{2}}, \quad K=\frac{c^{\mu / 2}(\mu / 2)^{m^{2 p+1}+1}}{\left(m^{4 p+1}+1\right) !} \frac{\left(c^{*}\right)^{m^{2 p+1}+1}}{\left(\log \left(k \tau_{1}\right)\right)^{2 m^{4 p+1}+2}}, p=\left[\frac{\log \left(6 k \tau_{1}\right)}{\log 2}\right]+2$,

(where [.] denotes the integral part). Put $r^{*}=\max \left\{M_{0}, M_{0}^{4 / \mu}\right\}$. From (11) we deduce that

$$
c \cdot c^{*} \frac{\left(\tau_{1} r\right)^{\mu / 2}}{\left(\log \left(k \tau_{1}\right) \log r\right)^{2}}>r^{\mu / 4} \geq M_{0}
$$

for $r \geq r^{*}$.

By Lemma 4, there exists $r_{0} \in\left[r^{*}, T^{-1}\left(T^{m}\left(r^{*}\right)\right)\right]$ such that

$$
\tau_{2} T\left(r_{0}\right) \leq T\left(\tau_{1} r_{0}\right)
$$

Put $r_{1}=r_{0} / k, R_{1}=\tau_{1} r_{0}$, then

$$
\frac{12}{\log k} \log \frac{2 R_{1}}{r_{1}} T\left(k r_{1}\right) \leq T\left(R_{1}\right),
$$

and

$$
12 T\left(r_{1}\right) \leq \frac{12}{\log k} \log \frac{2 R_{1}}{r_{1}} T\left(k r_{1}\right) \leq T\left(R_{1}\right)
$$


By (8), (9), (14), (15) and Lemma 2, there exists $z_{0}$ lying in $r_{1}<|z|<2 R_{1}$ such that in the disk

$$
\Gamma_{0}:\left|z-z_{0}\right|<\frac{4 \pi}{\log r^{*}}\left|z_{0}\right|
$$

$f$ takes every complex value $a$ at least

$$
n_{0}=c^{*} \frac{T\left(R_{1}\right)}{\left(\log r^{*}\right)^{2}\left(\log \left(k \tau_{1}\right)^{2}\right)}
$$

times except for those complex values which can be contained in two spherical disks $\gamma_{0}^{\prime}$ and $\gamma_{0}^{\prime \prime}$ with radius $e^{-n_{0}}$, that is, $\Gamma_{0}$ is a filling disk of $f(z)$. Obviously,

$$
n_{0} \geq \frac{c^{*}}{\left(\log k \tau_{1}\right)^{2}} \frac{T\left(1 / 2\left|z_{0}\right|\right)}{\left(\log \left(k\left|z_{0}\right|\right)\right)^{2}} \geq\left(\left|z_{0}\right|\right)^{\mu-\varepsilon\left(\left|z_{0}\right|\right)},
$$

where $\varepsilon(r)>0$, and $\varepsilon(r) \rightarrow 0$ as $r \rightarrow \infty$. It can be easily verified from (8) that

$$
\Gamma_{0} \subset\left\{z\left|\frac{1}{2 k} r^{*}<\right| z \mid<3 \tau_{1} T^{-1}\left(T^{m}\left(r^{*}\right)\right)\right\} .
$$

Put $t_{j}=T^{-1}\left(T^{m^{j}}\left(r^{*}\right)\right)$. It is obvious that $t_{0}=r^{*},\left\{t_{j}\right\}$ is an increasing sequence and $t_{j} \rightarrow \infty$. So the sequence of annuli $A_{j}=\left\{z\left|t_{j} / 2 k<\right| z \mid<3 \tau_{1} t_{j+1}\right\}$ tends to infinity as $j \rightarrow \infty$ and $\Gamma_{0} \subset A_{0}$. By $T\left(t_{j+1}\right)=T^{m}\left(t_{j}\right),(7)$ and (10) we get

$$
T\left(t_{j+2}\right)=T^{m}\left(t_{j+1}\right)>T^{2 \alpha}\left(t_{j+1}\right)>T\left(2 t_{j+1}\right) .
$$

It follows that $t_{j+2}>2 t_{j+1}$, so $t_{j+p}>2^{p-1} t_{j+1}$, and thus $t_{j+p}>6 k \tau_{1} t_{j+1}$. Therefore,

$$
A_{j} \cap A_{j+p}=\emptyset \quad(j=0,1,2, \cdots) .
$$

Next we prove that there is at least one in five annuli $A_{p}, A_{2 p}, A_{3 p}, A_{4 p}, A_{5 p}$ which does not meet $\gamma_{0}^{\prime} \cup \gamma_{0}^{\prime \prime}$. Assume $\gamma_{0}^{\prime}$ (or $\left.\gamma_{0}^{\prime \prime}\right)$ meet both $A_{j p}$ and $A_{(j+2) p}(j \in\{1,2,3\}$ ). Then we have

$$
e^{-n_{0}} \geq \frac{3 \tau_{1} t_{(j+1) p+1}-\frac{1}{2 k} t_{(j+1) p}}{\sqrt{1+9 \tau_{1}^{2} t_{(j+1) p+1}^{2}} \sqrt{1+\frac{1}{4 k^{2}} t_{(j+1) p}^{2}}} \geq \frac{c}{t_{(j+1) p+1}},
$$

where $c>0$ is the constant in (12). This means

$$
T^{m^{(j+1) p+1}}\left(r^{*}\right) \geq T\left(c e^{n_{0}}\right) .
$$


On the other hand, by (10) and (13) we have

$$
c e^{n_{0}}>c n_{0}>c \cdot c^{*} \frac{\left(\tau_{1} r^{*}\right)^{\mu / 2}}{\left(\log \left(k \tau_{1}\right) \log r^{*}\right)^{2}} \geq M_{0},
$$

and hence

$$
T\left(c e^{n_{0}}\right)>c^{\mu / 2} e^{(\mu / 2) n_{0}}>c^{\mu / 2} \frac{(\mu / 2)^{m^{(j+1) p+1}+1}}{\left(m^{(j+1) p+1}+1\right) !} n_{0}^{m^{(j+1) p+1}+1}>K \frac{T^{m^{(j+1) p+1}+1}\left(r^{*}\right)}{\left(\log r^{*}\right)^{2 m^{4 p+1}+2}}
$$

where $K>0$ is the constant in (12). By (17) and (18) we have

$$
T\left(r^{*}\right)<\frac{1}{K}\left(\log r^{*}\right)^{2 m^{4 p+1}+2} .
$$

This contradicts (9). Therefore, $\gamma_{0}^{\prime}$ (or $\gamma_{0}^{\prime \prime}$ ) can not meet both $A_{j p}$ and $A_{(j+2) p}(j \in\{1,2,3\})$. It follows immediately that there exists at least one in five annuli $A_{p}, A_{2 p}, A_{3 p}, A_{4 p}, A_{5 p}$ which does not meet $\gamma_{0}^{\prime}$ or $\gamma_{0}^{\prime \prime}$. Denote this annulus by $A_{0}^{1}$. So $f\left(\Gamma_{0}\right) \supset A_{0}^{1}$.

By the same discussion, we can deduce that there exists a filling disk $\Gamma_{1} \subset A_{0}^{1}$ and an annulus $A_{0}^{2} \in\left\{A_{j} \mid j \in \mathbf{N}\right\}$ such that $f\left(\Gamma_{1}\right) \supset A_{0}^{2}$. Repeating this construction, we obtain a sequence of filling disks $\Gamma_{j}$ such that

$$
f\left(\Gamma_{j}\right) \supset \overline{\Gamma_{j+1}}, \Gamma_{j} \rightarrow \infty(j \rightarrow \infty) .
$$

Denote the centre of $\Gamma_{j}$ by $z_{j}$. From (16) we know that each limiting point of $\left\{\arg z_{j} \mid\right.$ $j=1,2, \cdots\}$ is a Borel direction of order at least $\mu$ (see [5]). It follows (19) that there is a sequence of domains $B_{j} \subset A_{0}$ such that $f^{j-1}\left(B_{j}\right)=\Gamma_{j}$ and $\Gamma_{0} \supset B_{j} \supset \overline{B_{j+1}}$.

Now, we prove $\left(\bigcap_{j=1}^{\infty} \overline{B_{j}}\right) \cap J(f) \neq \emptyset$ : Otherwise, there exists a natural number $j_{0}$ such that $B_{j} \subset F(f)$ when $j \geq j_{0}$. Since $\Gamma_{0}$ is a filling disk, we have $f^{j}\left(B_{j}\right)=$ $f\left(\Gamma_{j}\right) \supset \overline{\mathbf{C}} \backslash\left(\gamma_{j}^{\prime} \cup \gamma_{j}^{\prime \prime}\right)$ (where $\gamma_{j}^{\prime}$ and $\gamma_{j}^{\prime \prime}$ are two spherical disks each with radius $e^{-n_{j}}$ and $n_{j} \rightarrow \infty$ as $\left.j \rightarrow \infty\right)$, so $J(f) \subset \gamma_{j}^{\prime} \cup \gamma_{j}^{\prime \prime}$ when $j \geq j_{0}$. This implies $J(f)$ contains at most two points. This is a contradiction [2].

For a point $a \in\left(\bigcap_{j=1}^{\infty} \overline{B_{j}}\right) \cap J(f)$, we have $a \in I(f)$ and each limiting direction of $O^{+}(a)$ is a Borel direction of order at least $\mu$. The proof of Theorem 3 is complete.

\section{REFERENCES}

[1] I.N. Baker, 'Infinite limits in the iteration of entire functions', Ergodic Theory Dynamical Systems 8 (1988), 503-507. 
[2] W. Bergweiler, 'Iteration of meromorphic functions', Bull. Amer. Math. Soc. 29 (1993), 151-188.

[3] A.E. Eremenko, 'On the iteration of entire functions', in Dynamical System and Ergodic Theory, Banach Center Publications 23 (Polish Scientific Publishers, Warsaw, 1989), pp. 339-345.

[4] W.K. Hayman, 'Angular value distribution of power series with gaps', Proc. London Math. Soc. 24 (1972), 590-624.

[5] L. Yang, Value distribution theory (Springer-Verlag, Berlin, Heidelberg, New York; Science Press, Beijing, 1993).

Center for Mathematics

China University of Mining and Technology (Beijing)

Xueyuan Road, Ding 11

Beijing 100083

Peoples Republic of China

e-mail: qjy@mail.cumtb.edu.en
Academy of Mathematics and System Sciences

Chinese Academy of Sciences

Beijing 100080

Peoples Republic of China 